\title{
BMJ Open Advanced chronic liver disease in the last year of life: a mixed methods study to understand how care in a specialist liver unit could be improved
}

\author{
Joseph Low, ${ }^{1}$ Sarah Davis, ${ }^{1}$ Victoria Vickerstaff, ${ }^{1}$ Lynda Greenslade, ${ }^{2}$ \\ Katherine Hopkins, ${ }^{3}$ Andrew Langford, ${ }^{4}$ Aileen Marshall, ${ }^{2}$ Douglas Thorburn, ${ }^{2}$ \\ Louise Jones ${ }^{1}$
}

To cite: Low J, Davis S, Vickerstaff V, et al. Advanced chronic liver disease in the last year of life: a mixed methods study to understand how care in a specialist liver unit could be improved. BMJ Open 2017;7:e016887. doi:10.1136/ bmjopen-2017-016887

- Prepublication history and additional material are available. To view these files, please visit the journal online (http://dx.doi org/10.1136/bmjopen-2017016887).

Received 17 March 2017 Revised 27 June 2017 Accepted 14 July 2017

\section{(a) CrossMark}

${ }^{1}$ Marie Curie Palliative Care Research Department, Division of Psychiatry, University College London, London, UK

${ }^{2}$ Sheila Sherlock Liver Unit, Royal Free Hospital, University College London Institute of Liver and Digestive Health, UCL Royal Free Campus, London, UK ${ }^{3}$ Department of Palliative Care, Royal Free London NHS Foundation Trust, London, UK ${ }^{4}$ British Liver Trust, Bournemouth, UK

Correspondence to Dr Joseph Low; joseph.low@ucl.ac.uk

\section{ABSTRACT}

Objective To identify the limitations in palliative care provision in the last year of life for people with liver cirrhosis and potential barriers to and enablers of palliative care.

Design Mixed methods, including a retrospective case note review, qualitative focus groups and individual interviews.

Setting A tertiary referral liver centre in the south of England (UK).

Participants Purposively selected case notes of 30 people with cirrhosis who attended the tertiary referral liver centre and died during an 18-month period; a purposive sample of 22 liver health professionals who participated in either focus groups or individual interviews. Primary and secondary outcomes Data collected from case notes included hospital admissions, documented discussions of prognosis and palliative care provision. Qualitative methods explored management of people with cirrhosis, and barriers to and enablers of palliative care.

Results Participants had high rates of hospital admissions and symptom burden. Clinicians rarely discussed prognosis or future care preferences; they lacked the skills and confidence to initiate discussions. Palliative care provision occurred late because clinicians were reluctant to refer due to their perception that reduced liver function is reversible, poor understanding of the potential of a palliative approach; palliative care was perceived negatively by patients and families.

Conclusions People dying with cirrhosis have unpredictable trajectories, but share a common pathway of frequent admissions and worsening symptoms as death approaches. The use of clinical tools to identify the point of irreversible deterioration and joint working between liver services and palliative care may improve care for people with cirrhosis.

\section{BACKGROUND}

Advanced chronic liver disease (cirrhosis) is a growing international public health problem and often affects people of working age. ${ }^{1}$ It is the third most common cause of premature death in the $\mathrm{UK}^{2}$; more people are affected
Strengths and limitations of this study

- The study is the first to look specifically at how care is provided to people with advanced liver disease in the last year of life, with the aim of identifying barriers that limit a palliative approach to care.

- A mixed methods design enables exploration from different perspectives of the structural difficulties to providing end of life care to people with advanced liver disease.

- Findings suggest pragmatic ways that supportive and end of life care can be improved for people with advanced liver disease.

- As this study was conducted in one tertiary liver unit in the south of England, the findings may not be generalised to other healthcare settings.

- The retrospective nature of the case note data limits the interpretation of the quantitative findings.

by liver disease with the increases in alcohol consumption, viral hepatitis and obesity. ${ }^{3}$ Most people dying from liver disease are not suitable for liver transplantation and, of those who are suitable, $20 \%$ will die before a donor becomes available. ${ }^{4}$ Living with cirrhosis may involve considerable symptom burden, and when liver failure ensues the prognosis is poor. Death may occur either after a long period of decline with a fluctuant clinical picture, or may be sudden and unanticipated. In most cases, death from cirrhosis occurs in hospital. ${ }^{5}$

People with cirrhosis have supportive and palliative care needs. ${ }^{6-9}$ Liver professionals acknowledge they have a role to play in this aspect of care, ${ }^{10}{ }^{11}$ but feel that their skills are limited and may be inadequate to offer an effective palliative approach. ${ }^{10}{ }^{12}$ Referrals to specialist palliative care (SPC) may be necessary but palliative care provision is limited, ${ }^{13}$ and knowledge of prescribing in liver failure 
is needed. Shared care, defined as using the skills and knowledge of many health professionals who share joint responsibility for an individual's care, may be useful. ${ }^{14}$ Palliative care offered in parallel with optimised specialist and generalist care may benefit people with advanced cirrhosis. ${ }^{15}$ One difficulty is knowing the appropriate time to make referrals and begin shared care. ${ }^{10}$ Further data to understand how different specialities such as liver and palliative care services can work together may be helpful. ${ }^{14}$ In this paper, we report what we have learnt from exploring practice in a tertiary treatment centre for liver disease in north London, UK. We used mixed methodology, guided by Rapid Participatory Appraisal in which data collected from different sources relating to a specific healthcare provider are combined to describe both the service structure and potential care improvements in a specific health locality. ${ }^{16}$ Using mixed methods, we hoped to gain greater understanding of the limitations in the provision of palliative care for people with cirrhosis in the last year of life, and explore the reasons behind these limitations. This approach is commonly used in health service research to understand the complexity of healthcare. $^{17}$

We conducted a case note review, focus groups and qualitative interviews to explore:

1. how healthcare in liver services is provided in the last year of life to people with advanced liver disease (cirrhosis) from any cause to identify limitations in palliative care provision;

2. challenges in providing palliative care provision in liver care and how this provision might be improved in hepatology.

\section{METHOD}

A mixed methods study, using a retrospective case note review, qualitative focus groups and individual interviews. Case note findings were used to quantify the types of healthcare inputs provided by the liver services to people in their last year of life and to identify potential limitations in and barriers to the palliative care provided and a shared approach to care. The qualitative data identified reasons underlying these limitations and barriers, and highlighted potential enablers to improving care in this context.

\section{Setting}

A tertiary referral liver transplant centre in north London UK, providing both a core diagnostic service for all conditions affecting the liver and long-term management of patients with all severities of liver disease.

\section{Procedure}

\section{Retrospective case note review}

As resources were limited, we purposively selected 30 people with cirrhosis from the 66 people who attended the tertiary referral liver centre and died between April 2010 and September 2011. We aimed to ensure that our sample represented the spectrum of people attending the centre and purposefully sampled according to age, gender and cause of liver failure.

We used a structured framework to extract data from patient records available from the centre for the 12-month period prior to death. We noted demographics, severity of liver disease at last admission, cause of cirrhosis, transplantation status, physical and psychological symptoms, and health service use in secondary care (inpatient admissions, hospital length of stay, intensive therapy unit (ITU) and liver-related procedures). We recorded documented evidence of discussions about prognosis and future preferences for care. We collected information on referrals to SPC, creation of care plans including evidence of advance care planning (ACP), resuscitation (do not attempt cardiopulmonary resuscitation (DNACPR)) status, preferred place of death and actual place of death. Data were extracted by the research nurse (SD) and inputted into Microsoft Excel.

\section{Qualitative data}

Both focus groups and semistructured interviews were used to capture as many views of healthcare professionals as possible. All potential participants were first identified by a clinician (LG). The research nurse (SD) then contacted these participants face to face, by telephone or by email. Participants were given information about the study, outlining the role of the research team, and gave written consent prior to data collection. All participants took part either in one focus group or a semistructured interview (between July 2013 and May 2014), which were conducted in the liver centre.

\section{Focus groups}

Focus groups were used as a pragmatic method of gathering larger numbers of people and using the group dynamic to generate discussion about care at the end of life in cirrhosis. ${ }^{18} 19$ Purposive sampling was used to ensure the views of those at all levels of the liver team across the disciplines (doctors, nurses and allied health professionals) were captured. Three focus groups were organised (each lasting 45-60 min) and led by a research nurse (SD-Master's degree qualification and 6 years of qualitative research experience), with an observer (JL-senior health researcher with a $\mathrm{PhD}$ and 20 years of experience in qualitative/mixed methods research) taking field notes and cofacilitating.

\section{Topic guide}

To guide discussions, a topic guide (online supplementary appendix 1) was developed by the members of research team (JL, SD, AM, DT, LG, KH and LJ) covering: challenges of providing care to people in the last year of life; their perception of patient and family understanding of liver disease; discussing prognosis and future care preferences; improving palliative care. This guide was developed pragmatically in the context of liver disease, guided by the principles of palliative 
Table 1 Patient demographics and clinical characteristics

Characteristic n (\%) (N=30)

Demographics

Age

$\begin{array}{lc}\text { Mean (SD) } & 58(11) \\ \text { Min-max } & 25-79 \\ \text { Gender } & \\ \text { Male } & 20(67)\end{array}$

Female

$10(33)$

Ethnicity $(n=24)$

$\begin{array}{lc}\text { White British } & 14(58) \\ \text { Black African } & 2(8) \\ \text { Asian } & 4(17) \\ \text { Other } & 4(17)\end{array}$

Relationship status $(n=26)$

$\begin{array}{lc}\text { Married } & 14(54) \\ \text { Divorced } & 5(19) \\ \text { Partner (previously divorced) } & 6(23) \\ \text { Widowed } & 1(4)\end{array}$

Living arrangements $(\mathrm{n}=28)$

$\begin{array}{ll}\text { With wife/partner } & 8(35) \\ \text { With wife and children } & 2(9) \\ \text { With children } & 3(13) \\ \text { With friends } & 2(9) \\ \text { Alone } & 5(22) \\ \text { Hostel } & 2(9) \\ \text { Hotel } & 1(4)\end{array}$

Clinical characteristics

Cause of cirrhosis

$\begin{array}{lr}\text { Alcoholic liver disease (ALD) } & 11(37) \\ \text { Hepatitis C (Hep C), ALD } & 3(10)\end{array}$

Hepatocellular carcinoma (HCC), Hep C,

ALD 1 (3)

HCC, Hepatitis B (Hep B), Hep C, ALD 1 (3)

Hep C 4 (13)

Hep C, HCC 3 (10)

Non-alcoholic steatohepatitis (NASH) 2 (7)

$\mathrm{NASH}, \mathrm{HCC}$

Other (primary biliary cirrhosis, antitrypsin)

$2(7)$

\begin{tabular}{ll} 
Previous transplant & $2(7)$ \\
On transplant list & $5(17)$ \\
\hline
\end{tabular}

care. $^{20}$ All focus groups were audiotaped and transcribed verbatim.

\section{Interviews}

Professionals unable to attend the focus groups, were invited to take part in semistructured individual interviews. Nine interviews were conducted by SD (lasting 18-70 min) using the topic guide, and were audiotaped and transcribed verbatim.

\section{DATA ANALYSIS \\ Retrospective case notes}

Descriptive statistics were used to describe hospital admissions and service use, documentation of prognostic discussions and preferences for future care, and palliative care provision. Data were summarised to highlight limitations in palliative care service provision.

\section{Qualitative data}

A framework approach was used to analyse the transcripts, ${ }^{21}$ which were first read independently by two researchers (JL and SD). Themes were identified, from which a coding system was developed and applied to the whole data set systematically. Any disagreements in coding were resolved by consensus. In organising the data into appropriate themes, Microsoft Excel was used. The researchers considered themes independently and met to discuss the themes identified and how they were linked together by contextual factors. Independent analysis ensured validity and reliability of the themes and links identified. Findings were also shared with our clinical partners (AM, LG, DT and $\mathrm{KH}$ ) in the research team to ensure that the findings were consistent with their experience of current clinical practice. These themes were used to explain the limitations in palliative care provision found in the case notes, and to identify barriers and enablers to future palliative care for people with cirrhosis.

\section{Ethical approval}

Ethical approval was sought, but deemed unnecessary by the NRES Committee London-West London \& GTAC (ref 14/LO/0799). NHS permission to conduct the clinical case note review and the qualitative interviews with liver health professionals was obtained from the Royal Free London Clinical Governance Lead for Hepatology and Palliative Care under the remit of health service improvement.

\section{RESULTS}

\section{Provision of healthcare in last year of life (case note} findings)

Demographics and clinical characteristics (table 1)

Patient demographics and clinical characteristics are provided in table 1 . Our sample was predominately male $(n=20,67 \%)$ with a median age of 59 years (IQR: 52-66; range 25-75), in which alcohol-related liver disease was the predominant diagnosis $(\mathrm{n}=16 ; 53 \%)$. A Model for End-stage Liver Disease (MELD) score gives an indication of short-term mortality, and is used to prioritise candidates on the orthotopic liver transplantation waiting list. In 
Table 2 Signs and symptoms during the last 3 months for the 30 patients

\begin{tabular}{|c|c|}
\hline Medical condition & n (\%) \\
\hline Pain & $24(80)$ \\
\hline Abdomen & $15(50)$ \\
\hline Back & $6(20)$ \\
\hline Legs & $4(13)$ \\
\hline Chest & $4(13)$ \\
\hline Ribs & $2(7)$ \\
\hline Ascites & $19(63)$ \\
\hline Distended abdomen & $12(40)$ \\
\hline Tense abdomen & $6(20)$ \\
\hline Encephalopathy & $19(63)$ \\
\hline Confusion & $11(37)$ \\
\hline Asterixis, hepatic flap & $10(33)$ \\
\hline Drowsiness & $8(27)$ \\
\hline Tremor & $5(17)$ \\
\hline Refusing treatment & $4(13)$ \\
\hline Agitation & $4(13)$ \\
\hline Distressed & $2(7)$ \\
\hline Crying, upset & $2(7)$ \\
\hline Aggressive & $2(7)$ \\
\hline Shouting/screaming & $2(7)$ \\
\hline Disorientated & $2(7)$ \\
\hline Bleeding & $12(40)$ \\
\hline Blood in faeces & $4(13)$ \\
\hline Blood in vomit & $3(10)$ \\
\hline Coffee ground vomit & $3(10)$ \\
\hline Bleeding from rectum & $4(13)$ \\
\hline Bruising under skin & $3(10)$ \\
\hline Bleeding from mouth/nose & $2(7)$ \\
\hline Bleeding from penis & $2(7)$ \\
\hline Blood in urine & $2(7)$ \\
\hline Peripheral oedema & $7(23)$ \\
\hline Sacrum, testicles, scrotum & $6(2)$ \\
\hline Legs, thighs & $3(10)$ \\
\hline Ankles, feet & $3(10)$ \\
\hline \multicolumn{2}{|l|}{ Skin other } \\
\hline Pruritus & $7(23)$ \\
\hline Rashes, erythema & $5(17)$ \\
\hline Cellulitis & $3(10)$ \\
\hline Fatigue & $2(40)$ \\
\hline Weakness (tiredness, lethargy) & $9(30)$ \\
\hline Sepsis & $8(27)$ \\
\hline Tachycardia & $4(13)$ \\
\hline Temperature, chills/rigours & $4(13)$ \\
\hline Psychological & $10(33)$ \\
\hline
\end{tabular}

Continued

\begin{tabular}{ll}
\hline Table 2 Continued & \\
\hline Medical condition & $\mathbf{n}(\%)$ \\
\hline Distressed, crying, upset & $4(13)$ \\
\hline Depressed & $4(13)$ \\
\hline Low mood & $3(10)$ \\
\hline Hallucinations & $3(10)$ \\
\hline Anxious & $2(7)$ \\
\hline Refusing treatments/ & $2(7)$ \\
observations & \\
\hline Insomnia & $2(7)$ \\
\hline Suicidal & $2(7)$ \\
\hline Digestive system & \\
\hline Anorexia & $11(37)$ \\
\hline Nausea & $11(37)$ \\
\hline Vomiting & $10(33)$ \\
\hline Incontinent of faeces & $10(33)$ \\
\hline Constipation & $6(20)$ \\
\hline Diarrhoea & $4(13)$ \\
\hline Respiratory & \\
\hline Shortness of breath & $17(57)$ \\
\hline Secretions & $4(13)$ \\
\hline Wheezy & $2(7)$ \\
\hline Urinary system & $8(27)$ \\
\hline Incontinent of urine & $8(27)$ \\
\hline Oliguria & $6(20)$ \\
\hline Other & $9(30)$ \\
\hline Confusion (variety of causes) & $4(13)$ \\
\hline Unsteady on feet/gait & $112(7)$ \\
\hline Dizzy and faint & \\
\hline Agitation & \\
\hline
\end{tabular}

23 cases where data were available at last admission, our sample had a median (IQR) MELD score of 23 (16.5-23), suggesting a $19.6 \%$ chance of dying in the next 3 months. ${ }^{22}$ Nineteen $(63 \%)$ people were not considered for liver transplant due to poor health, four were on the transplant waiting list and three had previously received a transplant. Eight (27\%) people with cirrhosis had been referred to the tertiary centre from 'out of area' hospitals either for a liver transplant assessment, or for specialist treatments, such as a transjugular intrahepatic portosystemic shunt (TIPS) or intensive management of bleeding.

The people in our sample were highly symptomatic (table 2). All were symptomatic 3 months before death, presenting with ascites $(n=22,73 \%)$, extensive peripheral oedema $(n=20,66 \%)$, severe fatigue and weakness $(n=20 ; 66 \%)$, and pain $(n=13 ; 43 \%)$. In the last month of life, our participants presented with an average of 14 physical symptoms per person. The majority $(\mathrm{n}=19,63 \%)$ were noted to have symptoms of 
hepatic encephalopathy, such as confusion, disorientation and agitation.

\section{Health service use in tertiary care}

Our sample of 30 had a median of three inpatient admissions (IQR: 2-5) per person in the last year of life, and a median length of stay of 31 days (IQR: 19-55). Seventeen $(57 \%)$ people were readmitted within 30 days of discharge. The frequency of admissions increased for most people $(n=29 ; 97 \%)$ in the last 3 months of life. Nineteen $(63 \%)$ people had more than one admission in the month before death, during which the median number of admissions was two (IQR: 1-3). Of the 78 admissions precipitated by cirrhotic complications, most required invasive procedures, such as paracentesis $(n=53 / 78,68 \%)$, blood transfusions $(n=13 / 78$, $17 \%)$, endoscopic variceal banding $(n=4 / 78,5 \%)$ and TIPS $(n=4 / 78,5 \%)$. During these admissions, each participant was seen in the last year of life by a mean of three different liver consultants (range 1-6). Nine $(30 \%)$ people were regularly reviewed by the hospital nurse-led patient-at-risk team (PART), to decide whether to escalate or de-escalate their treatment. Six $(20 \%)$ people with cirrhosis required treatment in the intensive therapy unit (ITU) during which three patients died.

Documentation of prognosis, future care discussions and palliative care provision

Liver consultants recorded having discussed prognosis mainly with family members $(\mathrm{n}=23,77 \%)$; discussions occurred very late, in $16(53 \%)$ cases $\leq 34$ days before the person died. Liver doctors recorded fewer discussions with patients about understanding of their disease or future care preferences $(n=16,53 \%)$, most of which occurred 1 month before death $(\mathrm{n}=9 / 16 ; 56 \%)$.

Although most people $(n=26 ; 67 \%)$ had a DNACPR decision recorded in their medical notes, this was completed by medical personnel, with limited consultation with either the person with cirrhosis $(n=5,17 \%)$ or their family member $(n=6,20 \%)$. In seven cases, the liver team had to be alerted about completing a DNACPR by other clinicians such as the PART team $(n=4)$, ITU $(n=2)$ or the emergency department $(n=1)$. Most people $(n=19,63 \%)$ had no discussions with doctors about their preferred place of care.

Most people with cirrhosis $(\mathrm{n}=21 ; 70 \%)$ were referred to SPC a median of 5 days before death. Twelve $(40 \%)$ people with cirrhosis documented as deteriorating were still receiving active treatment up until their death. For most people, death occurred in hospital $(\mathrm{n}=25 ; 83 \%)$, three died in ITU. The remaining five people died either at home $(n=3$; $10 \%)$ or in a hospice $(n=2 ; 7 \%)$. Only five people from the sample had clear discussions with health professionals about place of death; two of these died in the place of their choice (one at home and the other in hospital).
Challenges to and enablers of provision of palliative care

(qualitative data)

\section{Demographics of liver clinician sample}

Thirteen liver health professionals took part in three focus groups (FG; FG1: three doctors, two liver transplant nurses, a dietician and a pharmacist; FG2: three ward nurses and a healthcare assistant; FG3: two ward nurses). Nine health professionals took part in semistructured interviews (five doctors, two senior nurses in hepatology, a clinical nurse specialist in palliative care and an alcohol liaison nurse). No demographic information was collected for the liver clinician sample other than their discipline.

\section{Key findings}

Initial analysis illustrated that liver clinicians recognised that although their patients were in poor health, they did not address quality of life issues with them and palliative care options were only considered with patients who raised this topic themselves. Further analysis identified five emergent themes which illustrated why liver clinicians focused on reactive treatment for people at the expense of palliative care: unpredictable trajectory of liver disease, management of patient treatment expectations, clinician/patient perceptions of the palliative care role, poor continuity of care, perceived lack of skill and confidence.

\section{Unpredictable trajectory of liver disease}

The perceived ability of the liver to recover function made it difficult for doctors to estimate the point of irreversible liver decline, and so provided doctors with hope that trying different treatments would promote recovery, even with patients on the wards who were imminently dying. Nurses felt that part of this difficulty was the short periods that doctors spend with patients in contrast with the ward nurses, who provide continuous care and were confident in identifying those imminently dying. However, nurses considered that cessation of active treatment was the responsibility of doctors as main clinical decision makers.

We (ward nurses) have constant contact with patients... enables us to identify those patients who are both aware of their deterioration and want to die at home to be fast tracked to specialist palliative care. Ward nurse, Nurse focus group 1.

\section{Management of patient expectations}

Doctors' emphasis on active treatments is reinforced by their own perceptions of patients' treatment expectations. Part of this expectation may be reflected by the patients' younger ages, who doctors feel want life at all costs. Furthermore, as many patients are referred by secondary care 'out of area' (as illustrated in our case notes), clinicians perceive these patients see referral to the tertiary liver centre as a last chance to 'cure' their liver disease. This in turn, reinforces clinicians' focus on active treatments, at the expense of discussing prognostic issues.

We probably don't do enough of it (discuss future care preference), because most of the patients 
at a given time are not willing to engage with that question. The median age of patients is 53 , so we are not talking about an 80 year old who has lived their life to the full. We are talking about people who still want life. (Consultant hepatologist 1, interview)

Patients' unrealistic expectations, and their limited knowledge and understanding of their own disease, presented doctors with difficulties in managing these expectations and deciding what treatment options to pursue.

They (patients) are often referred extremely late, full of expectation only to be told there's nothing we can do. The difficulty is, what do you then do with that patient? Do you let them go back to the referring trust or secondary care, how do you know that they are going to get palliative care or the treatment that they need. (Consultant hepatologist 2, interview)

\section{Misunderstanding of palliative care}

Clinicians perceived that patients and their family members saw referral to palliative care negatively, as a move suggesting that clinicians had 'given up' on the patient. They felt that patients and families did not understand what palliative care could offer in terms of symptom control and psychosocial support, instead seeing palliative care as a service for people at the very end of life, as illustrated by this senior nurse.

We've got a patient on the ward whose family are very opposed to palliative care, but wanted active treatment. The patient has had repeated admissions, even if the family can only have her for another extra few months. The nurse tried to tell them it is not just the last weeks and hours (input from palliative care), it can be longer than that and the palliative team have a lot to offer you even now. (ward senior nurse, interview)

Although most liver clinicians saw a role for palliative care, the debate on its utilisation centred more on understanding when a referral to palliative care was considered appropriate. Most had very limited experience in working with palliative care and were unsure of the best time to refer. This was further compounded by the difficulty of estimating the point of irreversible liver deterioration and the lack of clinical tools and guidelines to support them with this process.

Would like to refer much earlier, but need to have an understanding at the point that specialist palliative care would like involvement. (Consultant hepatologist, Multi-disciplinary focus group)

\section{Poor continuity of care}

Liver clinicians felt the lack of adequate information systems and the rotation of medical staff (our case notes showed that each participant saw at least three liver consultants over the year), contributed to "poor continuity of care'. This lack of continuity is demonstrated when treatment plans agreed with one consultant can be changed by another consultant due to a lack of shared information.

This rotation of staff causes problems as some patients are treated and patched up, but come in under another consultant when readmitted and treatment happens again. However, the system does not allow for information to be exchanged about what exact changes have occurred in their condition. (Consultant hepatologist 3, interview)

\section{Perceived lack of skill and confidence}

Both doctors and nurses perceived they lacked skills and confidence in engaging in discussions about prognosis or palliative care with patients or family members. On liver wards, this was further compounded by a lack of private space to discuss sensitive topics.

\section{Enablers for improved palliative care}

Liver clinicians suggested strategies to enhance both continuity and integration of palliative care and liver services, such as joint liver and palliative care clinics for people with decompensated liver disease and multidisciplinary team case conferences to coordinate care and treatment for those patients frequently admitted. Such initiatives would enhance mutual understanding across specialities of liver-specific symptom management and the timing of referrals. To support liver clinicians in identifying patients suitable for early palliative care support, appropriate clinical tools with relevant guidelines need to be identified.

\section{DISCUSSION}

\section{Key summary}

Our findings reflect the complicated clinical picture surrounding the provision of care of people with cirrhosis in their last year of life. We demonstrate that patients have a high symptom burden, increasing number of admissions in their last 3 months of life and a focus on active, disease-directed treatments. As with previous studies, ${ }^{12}{ }^{13}$ we highlight poor palliative care provision, in which discussions about prognosis and resuscitation orders were only raised in the last few days of life and referrals to palliative care were made very close to death. We found that liver clinicians have difficulties in initiating discussions regarding prognosis, do not engage in parallel planning for potential deterioration as well as recovery and have a limited knowledge of palliative care.

Studies suggest that uncertainty is an important barrier to anticipatory care planning in advanced liver disease. ${ }^{12}$ Our qualitative data further illustrate how five key factors interact as barriers to palliative care. Although liver clinicians may wish to refer patients to palliative care earlier, active treatment is usually the de-facto choice unless patients themselves specifically raise the topic. The difficulty of identifying the point of irreversible liver 
deterioration, patients' expectations of finding a cure, together with liver clinicians' perceived lack of confidence and skills in addressing palliative care issues enabled a focus on active treatment. A further barrier is the lack of contact and experience that liver clinicians have of working with palliative care specialists. This prevents them from understanding what palliative care can offer, and prevents palliative care clinicians from establishing earlier contact with patients which might enable them to become familiar faces for patients and families. This culture of active treatment may stem from tertiary centres being seen as at the forefront of technical innovation.

\section{Clinical implications}

Our findings suggest that lack of knowledge about the role and potential benefits of palliative care may contribute to the late referral of liver patients to SPC. The formation of liver clinics specifically for people with decompensated liver failure, with joint input from liver and palliative care specialists is recommended. This may promote understanding across specialties, an integrated and timely approach to care, formulation of treatment plans and a reduction in unplanned inpatient admissions to the liver service. ${ }^{12}$ It may also improve symptom control and enable clinicians to engage in discussions about prognosis and future care preferences with patients and families at an earlier stage. Previous studies have shown that early referral to SPC may reduce the rates of expensive hospitalisation, especially in the last month of life. ${ }^{23}$ Such service developments could be explored, in line with guidance set by the 'End of life care good practice guide'. ${ }^{24}$ Consideration should be given to the care philosophy in a tertiary liver transplant centre, where many liver clinicians are reluctant to accept that active interventions have limited patient benefit. Qualitative data indicate that liver clinicians found it difficult to identify the point of irreversible liver deterioration; our case note findings suggest that inpatient admissions and symptoms increase in frequency in the last 3 months of life. The introduction of clinical tools such as the Supportive and Palliative Indicators Care Tool $^{25}$ may support clinicians to identify when is timely to refer to palliative care, such as the 'point of irreversible deterioration of liver function'.

\section{Strengths and limitations}

Our study explores care in advanced liver disease from different perspectives, but we accept our methodology limits the generalisability of our interpretation. Our case note data were retrospective and limited by the quality of recording in medical notes. Many in our case note sample were referred from other hospitals and did not include data recorded at these sites. Due to time constraints, we reviewed a purposive sample of case notes of those who died, so there is a potential for selection bias and error in the notes that were reviewed. Our case note sample only reflects patients who died during follow-up and not those who were still alive, or who had a transplant. This is important since these patients are also often recipients of palliative care. For our qualitative arm, the health professionals were recruited from one hospital site and due to both time constraints and the limited pool of participants available, it is possible that theme saturation was not fully achieved. We did not explore the views of close family members and informal carers in this study and may have missed important insights on experiences of living and dying with liver disease and how care might be improved. Our findings reflected practice in a tertiary liver transplant specialist unit in one country; while clinical issues are likely to be similar in other settings, organisational issues and person-centred attitudes will vary across other healthcare systems. Nevertheless, our exploratory findings do provide new insights into how care towards the end of life could be improved in people with cirrhosis, which deserve further exploration using more robust methodology.

Contributors JL, SD, W, LJ, LG and KH were responsible for the study concept and design; JL and SD were responsible for the acquisition of the data; JL, SD, VV, LJ, $\mathrm{DT}, \mathrm{AM}, \mathrm{LG}, \mathrm{KH}$ and $\mathrm{AL}$ were responsible for analysis or interpretation of the data; $\mathrm{JL}, \mathrm{SD}, \mathrm{V}$ and $\mathrm{LJ}$ drafted the initial manuscript; DT, AM, LG, KH and AL revised the manuscript critically for important intellectual content; all authors gave the final approval of the version to be published.

Funding This study received no specific grant from any funding agency in the public, commercial or not-for-profit sectors, but the research department responsible for this study is provided core funding by Marie Curie in order to conduct the study.

\section{Competing interests None declared.}

Ethics approval Ethical approval was sought, but deemed unnecessary by the NRES Committee London-West London \& GTAC (ref 14/L0/0799). NHS permission to conduct the clinical case note review and the qualitative interviews with liver health professionals was obtained from the Royal Free London Clinical Governance Lead for Hepatology and Palliative Care under the remit of health service improvement.

Provenance and peer review Not commissioned; externally peer reviewed.

Data sharing statement Participants did not provide consent for the transcripts to be released outside of the remit of this study.

Open Access This is an Open Access article distributed in accordance with the Creative Commons Attribution Non Commercial (CC BY-NC 4.0) license, which permits others to distribute, remix, adapt, build upon this work non-commercially, and license their derivative works on different terms, provided the original work is properly cited and the use is non-commercial. See: http://creativecommons.org/ licenses/by-nc/4.0/

(c) Article author(s) (or their employer(s) unless otherwise stated in the text of the article) 2017. All rights reserved. No commercial use is permitted unless otherwise expressly granted.

\section{REFERENCES}

1. Williams R. Global challenges in liver disease. Hepatology 2006;44:521-6.

2. Williams R, Aspinall R, Bellis M, et al. Addressing liver disease in the UK: a blueprint for attaining excellence in health care and reducing premature mortality from lifestyle issues of excess consumption of alcohol, obesity, and viral hepatitis. Lancet 2014;384:1953-97.

3. Right Care NHS. Liver disease: the NHS atlas of variation in healthcare for people with liver disease. 2013 http://www.rightcare. nhs.uk/index.php/atlas/liver-disease-nhs-atlas-of-variation-inhealthcare-for-people-with-liver-disease/ (accessed 22 Sep 2015).

4. Northup PG, Intagliata NM, Shah NL, et al. Excess mortality on the liver transplant waiting list: unintended policy consequences and Model for End-Stage Liver Disease (MELD) inflation. Hepatology 2015;61:285-91.

5. Effiong K, Osinowo A, Pring A, et al. Deaths from liver disease: implications for end of life care in England. National End of Life Care Intelligence Network, 2012. 
6. Roth K, Lynn J, Zhong Z, et al. Dying with end stage liver disease with cirrhosis: insights from SUPPORT. Study to understand prognoses and preferences for outcomes and risks of treatment. $J$ Am Geriatr Soc 2000;48(5 Suppl):S122-30.

7. Potosek J, Curry M, Buss M, et al. Integration of palliative care in end-stage liver disease and liver transplantation. J Palliat Med 2014;17:1271-7.

8. Sanchez W, Talwalkar JA. Palliative care for patients with end-stage liver disease ineligible for liver transplantation. Gastroenterol Clin North Am 2006;35:201-19.

9. Hansen L, Sasaki A, Zucker B. End-stage liver disease: challenges and practice implications. Nurs Clin North Am 2010;45:411-26.

10. Low J, Vickerstaff $\mathrm{V}$, Davis $\mathrm{S}$, et al. Palliative care for cirrhosis: a UK survey of health professionals' perceptions, current practice and future needs. Frontline Gastroenterology 2015.

11. Kendrick E. Getting it right: improving end of Life Care for people living with liver disease. Leicester: NHS Liver Care, National End of Life Care Programme, 2013. http://www.liver.nhs.uk/resources/end of_life_care/. (accessed 21 Mar 2013).

12. Kimbell B, Boyd K, Kendall M, et al. Managing uncertainty in advanced liver disease: a qualitative, multiperspective, serial interview study. BMJ Open 2015;5:e009241.

13. Poonja Z, Brisebois A, van Zanten SV, et al. Patients with cirrhosis and denied liver transplants rarely receive adequate palliative care or appropriate management. Clin Gastroenterol Hepatol 2014;12:692-8.

14. Luckett T, Phillips J, Agar M, et al. Elements of effective palliative care models: a rapid review. BMC Health Serv Res 2014;14:1-22.
15. Baumann AJ, Wheeler DS, James M, et al. Benefit of early palliative care intervention in end-stage liver disease patients awaiting liver transplantation. J Pain Symptom Manage 2015;50:882-6.

16. Fergus CJ, Chinn DJ, Murray SA. Assessing and improving outof-hours palliative care in a deprived community: a rapid appraisal study. Palliat Med 2010;24:493-500.

17. Creswell JW, Klassen AC, Plano Clark VL, et al. Best practices for mixed methods research in the health sciences. Bethesda (Maryland: National Institutes of Health, 2011:2094-103.

18. Kitzinger J. Qualitative research. Introducing focus groups. BMJ 1995;311:299.

19. Hudson P. Focus group interviews: a guide for palliative care researchers and clinicians. Int J Palliat Nurs 2003;9:202-7.

20. World Health Organization. Palliative care, 2007.

21. Ritchie J, Spencer L, O'Connor W. Carrying out qualitative analysis. Qualitative research practice: A guide for social science students and researchers. 2003:219-62.

22. Wiesner R, Edwards E, Freeman R, et al. Model for end-stage liver disease (MELD) and allocation of donor livers. Gastroenterology 2003;124:91-6.

23. Temel JS, Greer JA, Muzikansky A, et al. Early palliative care for patients with metastatic non-small-cell lung cancer. N Engl J Med 2010;363:733-42.

24. Healthcare for London. The end of life care good practice guide. 2010. http://www.londonhp.nhs.uk/wp-content/uploads/2011/03/ EOLC-good-practice-guide-plain-text.pdf.

25. Highet G, Crawford D, Murray SA, et al. Development and evaluation of the supportive and Palliative Care Indicators Tool (SPICT): a mixed-methods study. BMJ Support Palliat Care 2014;4:285-90. 